\title{
Diffusion tensor imaging demonstrates deviation of fibres in normal appearing white matter adjacent to a brain tumour
}

\author{
U C Wieshmann, M R Symms, G J M Parker, C A Clark, L Lemieux, G J Barker, \\ S D Shorvon
}

\begin{abstract}
the objective was to study fibre orientation in the cerebral white matter of a patient with a brain tumour using diffusion tensor imaging (DTI).

A patient with a mild left hemiparesis and a tumour in the right frontal lobe and 20 healthy volunteers were scanned with a DTI sequence. The scans were spatially normalised and the fibre orientation in the patient compared with the fibre orientation in normal controls.

DTI disclosed a change of the orientation of fibres in the patient compared with normal controls. In the normal appearing white matter adjacent to the tumour fibres deviated from the normal superior inferior orientation in the corona radiata by about $30^{\circ}$. This finding was consistent with a displacement by distant mass effect rather than a destruction of fibres, in agreement with the neurological examination.

In conclusion, DTI demonstrated a deviation of fibres in normal appearing white matter adjacent to a tumour. The technique will improve understanding of the effects of structural abnormalities on fibres. This will assist the interpretation of clinical findings and functional imaging studies and guide neurosurgical interventions.
\end{abstract}

(F Neurol Neurosurg Psychiatry 2000;68:501-503)

Keywords: diffusion tensor imaging; brain tumours

In the nervous system, myelinated tracts connect different brain regions together, and the brain with the spinal cord. Studying connections in normal and abnormal brains plays an important part in our understanding of brain function. Standard magnetic resonance ${ }^{1}$ and FLAIR $^{2}$ imaging techniques have been used to demonstrate tracts. However, information on the orientation of fibres within the tract is unobtainable with these methods. Diffusion imaging now provides an opportunity to study fibre orientation in vivo. In the white matter of the brain diffusion is directional (anisotropic) because water mol- ecules diffuse predominantly parallel to tracts. ${ }^{3}$ Diffusion tensor imaging (DTI), a new MRI technique, provides quantitative measures of the molecular motion of water in a three dimensional space $^{4}$ and can be used for accurate studies of fibre orientation in vivo. ${ }^{5}$ The principal eigenvector represents the principal direction of diffusion corresponding to the fibre tract axis. We used DTI in combination with spatial normalisation and a multiplanar representation of the principal eigenvector to demonstrate the directional organisation of human white matter in vivo ("tractography"). In this study we have investigated the fibre orientation in normal appearing white matter adjacent to a tumour.

\section{Methods}

We studied a 47 year old right handed man who developed partial epilepsy at the age of 38. On examination, he had only a mild paresis affecting the left leg (power 4/5) and impaired fine finger movements of the left side. Standard MRI with T1 weighted and T2 weighted sequences showed a large tumour in the right frontal lobe extending to the level of the corpus callosum. The long history of epilepsy and the appearance of the tumour on standard MRI were compatible with a low grade glioma.

\section{DIFFUSION TENSOR IMAGING VARIABLES}

We scanned the patient and 20 healthy volunteers (mean age 30 years) with a $1.5 \mathrm{~T} \mathrm{GE}$ scanner (GE, Milwaukee, USA, maximum gradient strength $=22 \mathrm{mT} / \mathrm{m}$, slew rate $=120$ $\mathrm{T} / \mathrm{m} / \mathrm{s}$ ). Diffusion tensor imaging was performed using an inversion recovery prepared single shot echo planar diffusion weighted imaging sequence $(\mathrm{TR}=5000 \mathrm{~ms}, \mathrm{TE}=78 \mathrm{~ms}$, $\mathrm{TI}=1835 \mathrm{~ms}$, FOV $24 \mathrm{~cm}$, acquisition matrix $96 \times 96$, reconstruction matrix $128 \times 128,5 \mathrm{~mm}$ slice thickness, $2 \mathrm{~b}$ values applied in seven noncolinear directions at 28 slice positions $\left.\left(b_{\max }=703 \mathrm{~s} / \mathrm{mm}^{2}\right)\right)$. From the measured signal on T2 weighted and diffusion weighted images, and the $b$ matrix calculated from the pulse sequence parameters, we estimated the effective diffusion tensor in each voxel. ${ }^{6}$ The diffusion tensor matrix was diagonalised to give the three eigenvalues and their associated 
eigenvectors. From this information the fractional anisotropy index and the mean diffusivity were derived on a pixel by pixel basis. The fractional anisotropy index is a scalar index of diffusion anisotropy which scales from 0 (isotropic medium) to 1 (maximum anisotropy) but does not provide information on the directions of diffusion. The eigenvector corresponding to the largest of the three eigenvalues is the principal eigenvector and represents the principal direction of diffusion at that point. The mean diffusivity is a measure of the magnitude of diffusion in $\mathrm{mm}^{2} / \mathrm{s}$ averaged over all directions. For spatial normalisation the nondiffusion weighted images were registered using a linear registration technique ${ }^{7}$ to a template which consisted of one of the controls in standard Talairach's space. ${ }^{8}$ The registration parameters were then used to transform the diffusion maps. As the diffusion eigenvectors are referenced with respect to the magnet and gradient axes, it was then necessary to apply the rotation part of the registration matrix to the eigenvectors to rotate them into the common space. We displayed both principal eigenvector and anisotropy ${ }^{9}$ and compared the appearance of fibres in the patient with the appearance of fibres in controls in corresponding anatomical positions. The pyramidal tract was defined as the fibres passing trough the posterior limb of the internal capsule. The angle between the AC-PC line and the pyramidal tract was measured on sagittal images.

\section{Results}

The tumour was located in Talairach's sector E and $\mathrm{F}$ in the expected position of the pyramidal tract. ${ }^{8}$ In the tumour the mean diffusivity was increased and the fractional anisotropy was reduced. (Mean diffusivity in white matter of normal control subjects: mean $=0.75 \times 10^{-3}$ $\mathrm{mm}^{2} / \mathrm{s}, \mathrm{SD}=0.05 \times 10^{-3} \mathrm{~mm}^{2} / \mathrm{s}$, in the patient $=$ $0.91 \times 10^{-3} \mathrm{~mm}^{2} / \mathrm{s}$, fractional anisotropy in control subjects: mean $=0.76, \mathrm{SD}=0.05$, in the patient: 0.29.) The abnormally high mean diffusivity values extended into the posterior limb of the internal capsule.

In the normal appearing white matter adjacent to the tumour (normal on visual inspection of $\mathrm{T} 1$ and $\mathrm{T} 2$ weighted images and within the normal range of mean diffusivity and fractional anisotropy) the orientation of

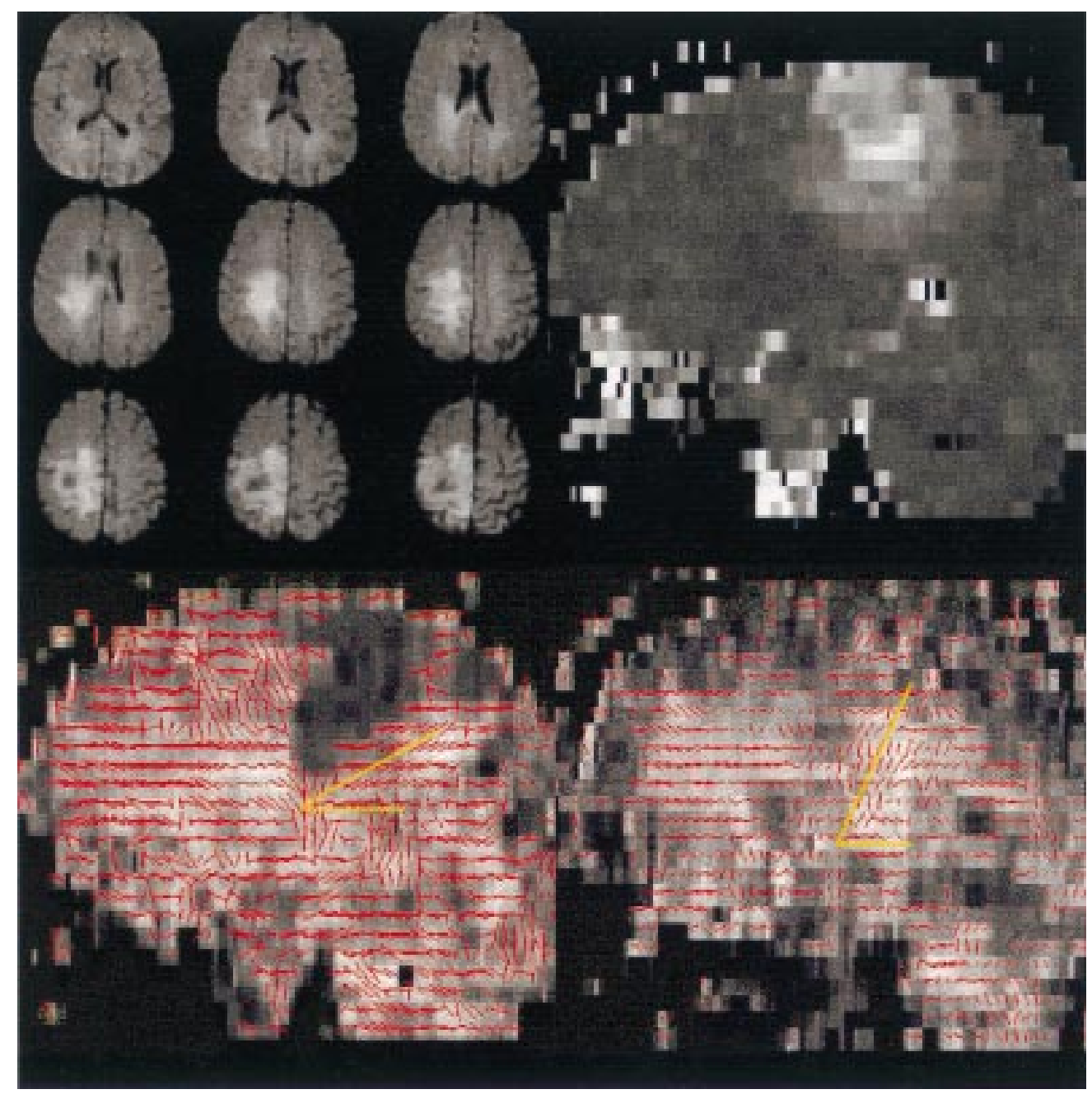

Fibre orientation in tumour and normal brain demonstrated with DTI. Top left: axial FLAIR images of the patient showing a tumour in the right central region. Top right: map of the mean diffusivity of the patient showing increased diffusion in the tumour. Bottom: map of the fractional anisotropy overlayed with the principal eigenvector (red bars) of the patient (left) and a control subject in the same position (right). In the normal appearing white matter adjacent the tumour the main fibre orientation (indicated by yellow bars) in the patient differs from the orientation in the brain of the control subject. 
the principal eigenvector differed from the orientation in corresponding regions on the contralateral side in the patient and from the orientation in the control subjects. In control subjects the angle between the AC-PC line and the pyramidal tract ranged from $50^{\circ}-80^{\circ}$, which was in keeping which other studies. ${ }^{8}$ In the patient the angle was about $35^{\circ}$, which implies a deviation of the principal eigenvector of about to $30^{\circ}$ (figure).

\section{Discussion}

We demonstrated an abnormal orientation of the principal eigenvector in normal appearing white matter adjacent to the tumour. The fact that mean diffusivity and fractional anisotropy were within normal limits in this region indicated that the tissue was as "densely packed" as normal brain tissue and retained a high degree of directional organisation. However, the deviation of the principal eigenvector in this region indicated that diffusion, although still directional, had taken a different orientation. Experimental studies have shown that water diffuses primarily parallel to fibres. ${ }^{10}$ Our findings therefore suggest a displacement (rather than a destruction) of fibres and showed that the tumour had a distant mass effect on normal appearing white matter. This information could not be obtained by other means. Our finding explains why the patient had only a mild neurological deficit despite the large tumour located in the expected position of the pyramidal tract.

With DTI the microstructural organisation in the brain can be investigated which may facilitate the detection of structural abnormalities in normal appearing white matter-for example, in multiple sclerosis. ${ }^{11}$ Displaying the principal eigenvector to study the direction of fibres allows the identification of abnormalities in the normal appearing white matter. This will improve the understanding of the organisation of fibres in the white matter of patients, not only in tumours but also in other abnormalities such as dysgenesis. The technique has the prospect of guiding neurosurgical interventions. ${ }^{12}$ Several different diffusion parameters and display techniques have been used to describe and visualise the directionality of white matter. ${ }^{13-16}$ We demonstrated a deviation of fibres in normal appearing white matter in a patient compared with spatially normalised control brains by visual (qualitative) means. In the future quantitative methods to assess displacement can be developed. The technique will assist the interpretation of neurological findings and functional imaging studies.

This research has been kindly funded by a European Community Fellowship (UCW), by the National Society for Epilepsy (MRS), the Brain Research Trust (CAC and MRS), and the Multiple Sclerosis Society of Great Britain and Northern Ireland (GJB and GJMP). We thank Dr B E Kendall, Dr J M Stevens, and Dr J S Duncan for their helpful comments.

1 Kuhn MJ, Johnson KA, Davis KR. Wallerian degeneration: evaluation with MR imaging. Radiology 1988;168:199-202.

2 Hajnal JV, De Coene B, Lewis PD, et al. High signal regions in normal white matter shown by heavily T2-weighted CSF nulled IR sequences. F Comput Assist Tomogr 1992;16:50613.

3 Moseley ME, Cohen Y, Kucharczyk J, et al. Diffusion weighted MR imaging of anisotropic water diffusion in cat central nervous system. Radiology 1990;176:439-45.

4 Basser PJ, Pierpaoli C. Microstructural and physiological features of tissues elucidated by quantitative-diffusiontensor MRI. F Magn Reson B 1996;111:209-19.

5 Hsu EW, Muzikant AL, Matulevicius SA, et al. Magnetic resonance myocardial fiber-orientation mapping with direct histological correlation. Am $\mathcal{f}$ Physiol 1998;274: H1627-34.

6 Basser PJ, Mattiello J, LeBihan D. Estimation of the effective self-diffusion-tensor from the NMR spin echo. $\mathcal{F}$ Magn Reson B 1994;103:247-54.

7 Woods RP, Cherry SR, Mazziotta JC. Rapid automated algorithm for aligning and reslicing PET images. $\mathcal{f}$ Comput Assist Tomogr 1992;16:620-33.

8 Talairach J, Tournoux P. Referentially oriented cerebral MRI anatomy. Stuttgart: Georg Thieme Verlag, 1993.

9 Parker GJM, Werring DJ, Barker GJ, et al. Multiplanar combined scalar and vector display for diffusion tensor bined scalar and vector display for diffusion tensor
information. Proceedings of the International Society for Maginformation. Proceedings of the International Society for Magnetic Resonance in Medicine,

10 Beaulieu C, Allen PS. Determinants of anisotropic water diffusion in nerves. Magn Reson Med 1994;31:394-400.

11 Werring DJ, Clark CA, Barker GJ, et al. Diffusion tensor imaging of lesions and normal-appearing white matter in multiple sclerosis. Neurology 1999;52:1626-32.

12 Alexander AL, Burr RB, McDonald J, et al. A technique for functional localization of the sensory motor cortex with diffusion anisotropy. Proceedings of the International Society for Magnetic Resonance in Medicine, 7th scientific meeting. Berkeley, CA: ISMRM, 1999:326.

13 Douek P, Turner R, Pekar J, et al. MR color mapping of myelin fiber orientation. $f$ Comput Assist Tomogr 1991;15: 923-9.

14 Makris N, Worth AJ, Sorensen AG, et al. Morphometry of in vivo human white matter association pathways with rol 1997;42:951-62.

15 Peled S, Gudbjartsson H, Westin CF, et al. Magnetic resonance imaging shows orientation and symmetry of white matter tracts. Brain Res 1998;780:27-33.

16 Mori S, Crain BJ, Chacko VP, et al. Three-dimensional tracking of axonal projections in the brain by magnetic resonance imaging. Ann Neurol 1999;45:265-9. 